\title{
Brain activity pattern changes after adaptive working memory training in multiple sclerosis
}

\author{
Laura Bonzano ${ }^{1,2}$ - Ludovico Pedullà ${ }^{3,4}$ - Matteo Pardini ${ }^{1,5}$ - Andrea Tacchino ${ }^{4}$ Paola Zaratin ${ }^{4}$. \\ Mario Alberto Battaglia ${ }^{6}$. Giampaolo Brichetto ${ }^{4}$. Marco Bove ${ }^{3,5}$
}

Published online: 30 October 2018

(C) The Author(s) 2018

\begin{abstract}
Cognitive impairment and related abnormal brain activity are common in people with multiple sclerosis (PwMS). Adaptive training based on working memory (WM) has been shown to ameliorate cognitive symptoms, although the effects at a neural level are unclear. The aim of this study was to expand the existing research on the effects of an adaptive WM rehabilitative intervention on brain functional activity in PwMS. A sample of eighteen PwMS performed an 8-week home-based cognitive rehabilitation treatment based on adaptive WM training. PwMS were assessed before and after treatment using a validated neuropsychological battery and undergoing an fMRI session while carrying out a cognitive task (i.e., Paced Visual Serial Addition Test - PVSAT). fMRI activations were compared to the activation pattern elicited by eighteen matched healthy subjects performing the same task. At baseline, we found abnormal brain activity during PVSAT in PwMS when compared to healthy subjects, with a pattern including several bilateral activation clusters. Following rehabilitation, PwMS improved cognitive performance, as evaluated by the neuropsychological battery, and showed a different activation map with clusters mainly located in the right cerebellum and in the left hemisphere. The only significant cluster in the right hemisphere was located in the inferior parietal lobule, and the BOLD signal extracted in this area significantly correlated with cognitive performance both before and after the treatment. We suggest that WM training can improve the cognitive performance and reduce the abnormal activation of PwMS by partially maintaining or even restoring brain cognitive function.
\end{abstract}

Keywords Cognitive rehabilitation $\cdot \mathrm{fMRI} \cdot$ Multiple sclerosis $\cdot$ Working memory $\cdot$ Adaptive training $\cdot$ Cognitive reserve

Laura Bonzano and Ludovico Pedullà contributed equally to this work.

Marco Bove

marco.bove@unige.it

1 Department of Neuroscience, Rehabilitation, Ophthalmology, Genetics, Maternal and Child Health, University of Genoa, Genoa, Italy

2 Magnetic Resonance Research Centre on Nervous System Diseases, University of Genoa, Genoa, Italy

3 Department of Experimental Medicine, Section of Human Physiology, University of Genoa, Viale Benedetto XV 3, 16132 Genoa, Italy

4 Italian Multiple Sclerosis Foundation, Scientific Research Area, Genoa, Italy

5 IRCCS Ospedale Policlinico San Martino, Genoa, Italy

6 Department of Life Science, University of Siena, Siena, Italy

\section{Introduction}

Cognitive impairment is a common symptom in multiple sclerosis (MS), with more than half of the people with MS (PwMS) showing cognitive deficits at formal neuropsychological testing (Amato et al. 2006a, b), especially impacting information processing speed and attentional abilities (DeLuca et al. 2015).

fMRI studies have explored cognitive processes in PwMS examining different functions, such as working memory, attention, and executive functions (Chiaravalloti and DeLuca 2008). In particular, the Paced Auditory Serial Addition Test (PASAT) (Audoin et al. 2005a, b; Forn et al. 2006; Mainero et al. 2004), the Paced Visual Serial Addition Test (PVSAT) (Bonzano et al. 2009), (i.e., the visual analogue of the PASAT (Nagels et al. 2005)), and the $N$-back task (Amann et al. 2011; Cader et al. 2006; Forn et al. 2007; Sweet et al. 2006) are among those more frequently used. Most studies conducted in both clinical and healthy populations have shown activations of frontoparietal brain areas involved in attention 
processing during the aforementioned cognitive tasks (Audoin et al. 2005a, b; Staffen et al. 2002).

However, fMRI studies have also demonstrated significantly altered patterns of cerebral activation in PwMS during the execution of different cognitive tasks (see for a review Chiaravalloti et al. 2015). In particular homologous region adaptation, local activation expansion and extra-region recruitment have been demonstrated to occur in MS. An activation likelihood estimation meta-analytic study (Kollndorfer et al. 2013) showed higher activation in the left ventrolateral prefrontal cortex and right premotor area in PwMS compared to healthy controls. In addition, it has been reported that PwMS can present enlarged patterns of bilateral cortical activations, possibly of compensatory nature (Bonzano et al. 2009; Pantano et al. 2006). Other studies have noted increased task-related fMRI activation in cognitively impaired PwMS related to worse performance on cognitive tasks, suggesting the occurrence of a process of maladaptive plasticity in these patients (Chiaravalloti et al. 2005; Hillary et al. 2003).

Recently, some studies have also provided evidence of improved cognitive performance, as well as improvements in everyday life activities, following cognitive rehabilitation (Amato et al. 2014; Chiaravalloti et al. 2013; Filippi et al. 2012; Mattioli et al. 2010). Among the different cognitive rehabilitation approaches, protocols based on working memory (WM) are of great interest because WM seems to be a mechanism linking cognitive performance to premorbid factors (e.g., verbal intelligence). In fact, WM has been shown to hold a mediating relationship between intellectual enrichment and long-term memory decline in individuals with MS (Sandry and Sumowski 2014). Moreover, WM capacity can be increased by training, as shown by behavioral (Kawashima et al. 2005; Uchida and Kawashima 2008) and neuroimaging (Olesen et al. 2004; Takeuchi et al. 2010) studies. In this regard, we have recently developed an application software for portable devices, named Cognitive Training Kit (COGNITRAcK), able to administer user-friendly and personalized treatments based on WM exercises that allow at-home interventions (Tacchino et al. 2015). Using this tool, we then evaluated the efficacy of adaptive (i.e., with fine tuning of exercises difficulty levels to the individual's performance) vs. nonadaptive WM training in cognitively impaired PwMS treated by means of COGNI-TRAcK (Pedullà et al. 2016). Our results demonstrated that the former approach has better outcomes, showing a significant performance improvement after the intervention only in the adaptive group even in tests evaluating non-trained cognitive domains, with positive effect maintained also after six months.

Based on these findings, we hypothesized that adaptive WM training would also induce changes in functional brain activation related to cognitive performance.

In this study, we aimed to test such hypothesis investigating the effects of adaptive WM training on functional brain activation in PwMS. To achieve this goal, we examined possible changes in brain activation obtained during a cognitive task before and after the training in a group of PwMS, compared to the brain activation pattern elicited by the same task in a group of healthy subjects (included here as a "reference pattern"). Specifically, a group of PwMS underwent a home-based treatment by means of COGNI-TRAcK, with automatic adjustment of tasks difficulty to individual's performance. Before and after the treatment, cognitive status was assessed using a validated MS-specific neuropsychological battery including PASAT. Brain activity was investigated by fMRI during the PVSAT. Healthy subjects underwent the same fMRI procedure once, and their cognitive performance was assessed with PASAT.

We expected to find alterations in task-related functional activation in PwMS at baseline, including an increase in the extent of activation of the brain areas used by healthy subjects as well as a recruitment of additional brain areas. We also hypothesized that the treatment might induce modifications in brain activity patterns, also based on brain efficiency in recruitment of resources, related to cognitive performance improvements.

\section{Materials and methods}

\section{Subjects}

The sample of this study was constituted of eighteen PwMS. The subjects were recruited among those complaining for cognitive disturbances during formal medical examination performed by a physician of the Italian Multiple Sclerosis Association rehabilitative center of Genoa.

The Rao's Brief Repeatable Battery of Neuropsychological Tests (BRB-NT) (Rao and The Cognitive Function Study Group of the National Multiple Sclerosis Association 1990) was used to assess cognitive functions at baseline.

Only patients who failed at least two tests of the BRB-NT, i.e., scoring 1.5 standard deviations (SD) below the normative values provided by Amato et al., 2006a, b, were considered cognitively impaired (Amato et al. 2010; Dackovic et al. 2016) and recruited for this study.

The BRB-NT assesses the most frequently impaired cognitive domains in PwMS and incorporates the following tests: Selective Reminding Test, for verbal memory acquisition (Selective Reminding Test-Long-Term Storage, SRT-LTS; Selective Reminding Test-Consistent Long Term Retrieval, SRT-CLTR) and delayed recall (Delayed Selective Reminding Test-Delayed, SRT-D); 10/36 Spatial Recall Test (SPART), for visual memory acquisition and delayed recall (Selective Reminding Test-Delayed, SRT-D); Paced Auditory Serial Addition Test in its variations at different stimuli presentation speed (PASAT-3 and PASAT-2) and Symbol Digit Modalities Test (SDMT), for sustained attention, concentration and information processing speed. We also 
added the Word List Generation (WLG) test for verbal fluency on semantic stimulus. The tests score was adjusted for years of formal education following the methodology described by Amato et al. 2006. The adjusted scores were used for all the analyses conducted in this study.

All the patients included in this study did not show any relapse in the 3 months prior enrolment nor were under corticosteroids treatments in that same time period. None of the subjects had positive history for major psychiatric disorders or for major medical comorbidities. Moreover, participants were not assuming benzodiazepines nor antidepressants during the study period, and did not present severe visual loss, dyscalculia, acalculia or MRI contraindication.

Among the enrolled patients (12 females and 6 males; mean age: $45.3 \pm 10.2$ years; mean education: $12.5 \pm$ 3.1 years), 12 were affected by a relapsing-remitting and 6 by a secondary-progressive MS course. In addition, 11 out of 18 patients were treated with disease-modifying drugs. The mean disease duration was $14.3 \pm 8.9$ years and median Expanded Disability Status Scale (EDSS) (Kurtzke 1983) score was 3.5, range: 1.0-6.5. In 12 out of 18 patients fatigue was perceived as a significantly impacting symptom, since they reported a score $>38$ at the Modified Fatigue Impact Scale (MFIS: mean score $=48.1 \pm 15.3$, range $=16-75$ ) (Flachenecker et al. 2002).

We also recruited eighteen age- and sex-matched healthy subjects (HS) as a control group (10 females and 8 males; age: mean $=41.6 \pm 1.3$ years, range: $38-43$ years). HS were not suffering from neurological or psychiatric disorder nor had personal or familiar history of mood disorders or substance abuse and showed no MRI contraindications.

The study was approved by the Ethics Committee of Azienda Ospedaliera San Martino, Genoa, Italy. All subjects provided a written informed consent according to the Declaration of Helsinki.

\section{Rehabilitation protocol}

The PwMS participating in the study executed an 8-week training consisting of five 30-min sessions a week. The sessions were self-administered at home by means of COGNI-TRAcK, with adaptive algorithms automatically adjusting the progression in task difficulty on the basis of the individual's performance. Three different types of WM exercises were executed every session (a visuospatial WM task, an "operation" $N$-back task and a "dual" $N$-back task) as described elsewhere (Tacchino et al. 2015). Briefly, in the visuospatial WM task patients had to remember a random sequence of visual stimuli presented one at a time in a grid-like interface and correctly reproduce it by touching the corresponding locations on the screen. In the "operation" $N$-back task, the stimuli consisted of pairs of numbers, 1 to 4, shown in a random sequence on the screen (e.g., $1+4)$. Participants were asked to memorize the sum of the two numbers presented (ranging from 2 to 8 ) and to push the button on the keyboard that corresponded to $N$ stimuli ago ( $N$-back rule). In the "dual" $N$-back task, the stimuli consisted of numbers, 1 to 4 , presented in a random sequence in one of four places on a line. Patients were asked to memorize the location and identity of the stimuli and their temporal order. Then, they had to push two buttons to indicate the identity and the location of the stimulus presented, according to the $N$-back rule. The adaptive paradigm adjusted the difficulty level of the exercises to the subject's performance in order to be more effective in improving cognitive functions in PwMS. Particularly, the exercises difficulty level increased by one step every time the subject performed an exercise correctly and decreased by one step if the exercise was done incorrectly for three times in a row. In details, based on a previous study (Takeuchi et al. 2010), in the first visuospatial WM task the level of difficulty was varied by changing the number of presented stimuli (minimum level: 4 stimuli). When participants indicated the correct location of all the stimuli in the presented order, it was regarded as a correct answer. In the two $N$-back tasks, the level of difficulty was varied by changing the value of $N$ (minimum level: $N=0$ ). Blocks were regarded as completed correctly when the participants made errors in less than $20 \%$ of the trials in the "operation" $N$-back task and less than $25 \%$ in the "dual" $N$ back task. In addition to the paradigm proposed by Takeuchi and colleagues, the inter-stimulus interval was also adjusted according to individual participant's performance. Thus, every main level, determined by the number of stimuli for the visuospatial WM task and by the $N$ parameter for the $N$-back tasks, was constituted by sub-levels with increased inter-stimulus rate. For each type of exercise, all PwMS started from the same low difficulty levels (namely, $2 \mathrm{~s}$ for the visuospatial WM task, $5 \mathrm{~s}$ for the "operational" $N$-back task and $7 \mathrm{~s}$ for the "dual" $N$-back task) in order to allow all patients with cognitive impairment to begin the training (Pedullà et al. 2016).

Adherence to treatment, calculated as the percentage of completed sessions on the total number of scheduled sessions, and mean results of each training session (i.e., difficulty level, accuracy of answers and, only for the $N$-back tasks, reaction time) were measured for each patient.

\section{Cognitive performance evaluation}

Before ("PRE session", i.e., baseline) and after ("POST session") the rehabilitation treatment, cognitive performance was evaluated in all PwMS with the BRB-NT in order to detect possible functional changes induced by the intervention. Version A and version B (Goretti et al. 2014) were adopted for the two sessions and randomly assigned so that half of the patients participating in the study received version A first while the other half received version B first. All tests were administered by a trained and certified cognitive examiner blinded to the session phase (POST vs. PRE). 
Furthermore, in order to assess the magnitude of these changes, the effect size of POST vs. PRE difference in PwMS cognitive performance was calculated by means of Cohen $\mathrm{d}$ method (Cohen 1988) and interpreted according to Sawilowsky descriptors $(\mathrm{d}=0.01$ : very small, $\mathrm{d}=0.20$ : small, $\mathrm{d}=0.50$ : medium, $\mathrm{d}=0.80$ : large, $\mathrm{d}=1.20$ : very large, or $\mathrm{d}=2.0$ : huge) (Sawilowsky 2009). Mean percentage change, calculated as ((POST score - PRE score) $/$ PRE score $) \times 100$, was computed for SDMT and PASAT-3, i.e., the tests investigating information processing speed which was shown to be the cognitive domain most widely affected by MS and the first cognitive deficit to emerge in PwMS (Van Schependom et al. 2015).

In addition, cognitive functions of HS were assessed with PASAT-3 only, in order to have a measure of HS cognitive performance to compare with PwMS performance. Indeed, this test was chosen among those included in the BRB-NT because it is the auditory analogue of PVSAT, that is the cognitive task all subjects (PwMS and HS) had to perform during the fMRI sessions.

\section{MRI acquisition}

MRI was acquired on a 1.5 T MR system (Signa HDxt General Electric, WI, USA) and included the following brain sequences: sagittal T1-weighted 3D spoiled gradient recalled (SPGR) imaging (slice thickness $=1.2 \mathrm{~mm}$; TR $=9.4 \mathrm{~ms}$; $\mathrm{TE}=3.9 \mathrm{~ms}$; flip angle $=8, \mathrm{FOV}=240 \times 240 \mathrm{~mm}$; matrix $=$ $256 \times 256$ ) for atrophy estimation, axial T2-weighted imaging ( slice thickness $=5 \mathrm{~mm} ; \mathrm{TR}=4000 \mathrm{~ms}$; TE $=104.6 \mathrm{~ms}$; $\mathrm{FOV}=240 \times 240 \mathrm{~mm}$; matrix $=256 \times 256$ ) as reference for fMRI, and axial single-shot spin-echo echoplanar imaging for $\mathrm{fMRI}$ (slice thickness $=5 \mathrm{~mm}$; TR $=3000 \mathrm{~ms}$; TE $=$ $60 \mathrm{~ms} ; \mathrm{FOV}=\mathrm{FOV}=240 \times 240 \mathrm{~mm}$; matrix $=64 \times 64)$. For each subject, the first 3 volumes of each fMRI run were discarded because of non-steady magnetization, and the remaining 80 volumes were used for the analysis. Within each run the subject performed either the PVSAT (i.e., active task) or a baseline visual task (i.e., control), according to a boxcar paradigm with two 30-s active task periods alternating with two 30-s control periods (Bonzano et al. 2009). During the PVSAT phase, Arabic digits (black numbers on a white background, range: 1-9) were presented via a projection mirror system one at a time and the subject was instructed to consecutively add pairs of numbers, such that each number was added consecutively to the one that immediately preceded it. The baseline visual task consisted of the presentation of a fixation cross. In both phases, each stimulus was presented for $1 \mathrm{~s}$ and followed by a blank screen for $2 \mathrm{~s}$. All participants familiarized with the PVSAT and the experimental protocol outside the scanner before the beginning of the imaging session. While inside the scanner they were instructed to calculate and not to spell the numbers or results to avoid artifactual brain activations. At the end of the fMRI session, they were asked to report the last sum obtained. As for PASAT, we adopted two different series of numbers randomly assigned to the subjects for the two experimental sessions.

\section{Structural MRI analysis}

For each patient and session, normalized brain volume (NBV) was estimated from T1-weighted 3D SPGR images with SIENAX (Smith et al. 2002). Segmentation of brain, including cerebellum, from non-brain tissue in the head was performed. The outer skull surface was estimated to normalize brain volume for skull size; all brain and skull images were registered to a standard space and a normalized brain volume estimate was obtained.

\section{Functional MRI analysis}

fMRI data processing was performed with Statistical Parametric Mapping software (SPM12, Wellcome Department of Imaging Neuroscience, London, UK) as described elsewhere (Friston et al. 1995). Briefly, each subject's time series were movement-corrected, normalized to the Montreal Neurological Institute (MNI) template brain image using a 12-parameter affine transformation and smoothed using an $8 \mathrm{~mm}$ full-width at half-maximum isotropic Gaussian kernel to increase the signal-to-noise ratio.

\section{Statistical analysis}

\section{Cognitive training and performance}

Changes in the training execution were investigated using an analysis of variance analysis (ANOVA) on mean difficulty level and accuracy of each session with TIME as within-subject factor (sessions 1 to 40) for all three types of exercises. Mean reaction time of each session was investigated only for the $N$-back tasks. Missing data of non-completed sessions were filled up with the values of the previous session, according to the "last observation carried forward" analysis (Altman 2009).

Concerning cognitive performance, data distribution was explored with the Shapiro-Wilk test to assess each BRB-NT test's score for normality. A paired t-test was used for each test of the battery in order to detect significant changes between the two sessions (PRE and POST) in the group of PwMS. Moreover, the PASAT-3 scores of PwMS in the two sessions were compared with HS performance by means of a t-test for independent samples.

\section{fMRI analysis}

A general linear model was used to identify the voxels with task-related signal changes at the individual level. Taskrelated $\mathrm{t}$ contrast images were created for every participant 
and were then introduced into a second-level random-effect analysis to allow for population inferences. One-sample t-tests were adopted to obtain the group activation maps (HS, PwMS in the PRE and POST sessions), with a height threshold of $p<0.05$ FWE-corrected and an extent threshold of 30 voxels per cluster. A paired t-test was then used to assess differences between the two sessions (PRE and POST) in the group of PwMS and t-tests for independent samples were used to assess differences in the PwMS in the single sessions separately with respect to HS. The analyses of statistical contrasts between sessions and groups were conducted with height threshold of $p<0.001$ uncorrected and minimum cluster size of 30 voxels.

\section{Correlation analysis}

Following the contrast analysis "PRE session $>$ POST session" we found significant clusters located in the left cingulate gyrus (BA 31), the right postcentral gyrus and inferior parietal lobule (BA 40). However, the right BA 40 was still significantly active in the POST session. To shed light on the role of this brain region on cognitive task performance, the first eigenvariate of the blood-oxygenation-level dependent (BOLD) fMRI signal was extracted in the corresponding significant cluster and correlated with PASAT performance adjusting for the NBV for the values obtained in the PRE and in the POST session, respectively.

\section{Results}

\section{Cognitive training and performance}

All patients completed the proposed protocol training along the 8 weeks without reporting any complaint and with a high adherence to treatment rate in all three exercise types $(89 \pm$ $13 \%, 87 \pm 13 \%$ and $85 \pm 13 \%$ for the visuospatial WM task, "operation" and "dual" $N$-back task, respectively).

Concerning the visuospatial WM task, the ANOVA showed a significant increase of the difficulty level reached throughout the 40 sessions $\left(F_{39,663}=18.34, p<0.0001\right)$ (Fig. 1a). Accuracy changed $\left(F_{39,663}=3.11, p<0.0001\right)$, but only between the first and the other sessions of the treatment, with higher values obtained in the first session due to the low starting difficulty levels. With regard to the "operation" $N$ back task, an increase of the difficulty level $\left(\mathrm{F}_{39,663}=19.24\right.$, $p<0.0001)$ and a decrease of the reaction time $\left(\mathrm{F}_{39,663}=\right.$ $12.84, \mathrm{p}<0.0001)$ were observed throughout the treatment (Fig. 1b). Again, accuracy decreased $\left(\mathrm{F}_{39,663}=2.86\right.$, $p<0.0001$ ) only between the first and the other sessions of the training. Similar results were found concerning the "dual" $N$-back task (Fig. 1c): the difficulty level increased throughout the 40 sessions $\left(\mathrm{F}_{39,663}=36.17, p<0.0001\right)$, whilst the reaction time decreased with TIME $\left(\mathrm{F}_{39,663}=19.91, \mathrm{p}<0.0001\right)$.
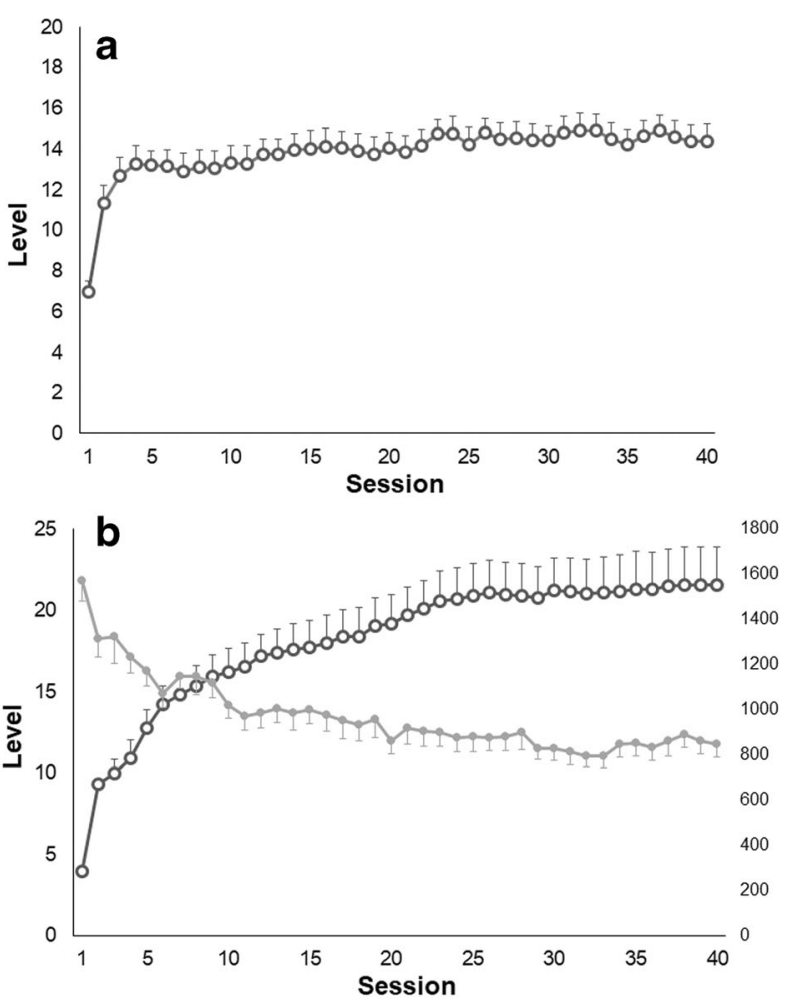

1800

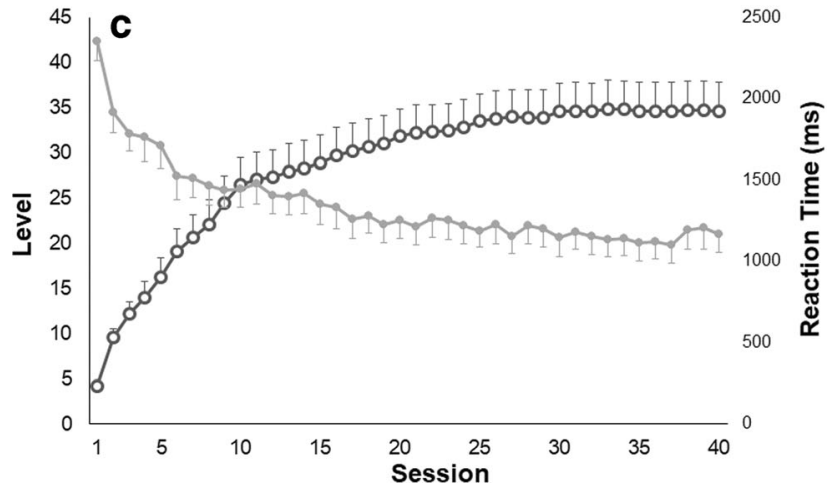

Fig. 1 Data showing the mean performance of subjects during the cognitive training: a mean difficulty level reached in each session in the visuospatial WM task; b mean difficulty level and reaction time of each session in the "operation" $N$-back task; c mean difficulty level and reaction time of each session in the "dual" $N$-back task. Error bars represent standard errors

In this case, accuracy changed slightly $\left(\mathrm{F}_{39,663}=1.45, p=\right.$ 0.04 ), decreasing between the first and the following sessions.

At baseline, all subjects obtained a score lower than $1.5 \mathrm{SD}$ below the normative data at two or more tests composing the BRB-NT. The median number of failed tests was 4 (range 28), with highest prevalence of failing observed at the SRT-LTS (72\% of patients), followed by SRT-CLTR (67\%), SRT-D and PASAT-2 (61\%). Half of the patients failed at PASAT-3 and SDMT, whilst lower percentages were observed at SPART $(44 \%)$ and SPART-D (17\%). None of the patients failed at the WLG.Shapiro-Wilk test showed that all tests' results were normally distributed, allowing the use of parametric statistics. 
The WM training was effective in improving the cognitive status of PwMS, as shown by their performance at the BRBNT. In fact, PwMS showed significantly higher scores at all the tests in the POST session than in the PRE session (Table 1). Effect size was large or very large for all tests (SRT-LTS: $\mathrm{d}=1.64$, SRT-CLTR: $\mathrm{d}=1.31$, SPART: $\mathrm{d}=1.03$, PASAT-3: $\mathrm{d}=1.34$, PASAT-2: $\mathrm{d}=1.48$, SRT-D: $\mathrm{d}=1.84$, SPART-D: $d=1.03$, WLG: $d=0.99)$, except for SDMT which showed medium effect size $(d=0.76)$. Mean percentage POST vs. PRE change was $20.73 \%$ for SDMT and $60.01 \%$ for PASAT-3.

\section{Brain activation during PVSAT}

The coordinates of the significant peaks of activation associated to PVSAT in the different groups are reported in Table 2. As also displayed in Fig. 2, the group activation map obtained for HS primarily included clusters located in the left cingulate gyrus (Brodmann's area (BA) 32), precuneus (BA 7), inferior parietal lobule (BA 40), precentral and middle frontal gyri (BA 6), and in the right cerebellum. The pattern of activation obtained in the PwMS group before cognitive rehabilitation (PRE session) includes bilateral activation clusters in the precuneus (BA 7), inferior parietal lobule (BA 40), and cerebellum, and clusters in the left BA 6 , and in the right postcentral gyrus (BA 2) and insula (BA 13) (Fig. 3a).

After cognitive rehabilitation (POST session), PwMS showed a different activation map (Fig. 3b), with clusters mainly located in the right cerebellum and in the left hemisphere: precuneus and superior parietal lobule (BA 7), precentral and superior frontal gyri (BA 6). The only

Table 1 Scores obtained by PwMS at the BRB-NT before (PRE) and after (POST) the rehabilitative intervention

\begin{tabular}{lllll}
\hline Test & PRE mean (SD) & POST mean (SD) & $p$ value & $\mathrm{t}$ \\
\hline SRT LTS & $24.74(7.51)$ & $41.35(12.25)$ & 0.000003 & 6.80 \\
SRT CLTR & $16.18(8.91)$ & $33.03(15.81)$ & 0.000184 & 4.75 \\
SPART & $14.46(4.25)$ & $19.27(5.07)$ & 0.001351 & 3.83 \\
SDMT & $39.96(10.33)$ & $48.24(11.40)$ & 0.000008 & 6.28 \\
PASAT-3 & $27.70(12.77)$ & $44.32(12.06)$ & 0.000000 & 8.16 \\
PASAT-2 & $18.97(9.02)$ & $33.02(9.99)$ & 0.000006 & 6.48 \\
SRT-D & $5.43(1.72)$ & $8.97(2.12)$ & 0.000004 & 6.62 \\
SPART-D & $4.56(1.08)$ & $6.31(2.13)$ & 0.003348 & 3.41 \\
WLG & $38.94(5.72)$ & $46.89(9.78)$ & 0.000063 & 5.26 \\
\hline
\end{tabular}

Student's t test values are reported

SRT-LTS, Selective Reminding Test-Long Term Storage; SRT-CLTR, Selective Reminding Test-Consistent Long-Term Retrieval; SPART, 10/ 36 Spatial Recall Test; SDMT, Symbol Digit Modalities Test; PASAT-3/ -2, Paced Auditory Serial Addition Test; $S R T-D$, Selective Reminding Test-Delayed; SPART-D, 10/36 Spatial Recall Test-Delayed; $W L G$, Word List Generation significant cluster in the right hemisphere was located in the inferior parietal lobule (BA 40).

As reported in Table 3, the contrast analysis between sessions (PRE and POST) in the PwMS group showed that the left cingulate gyrus (BA 31), the right postcentral gyrus and the right inferior parietal lobule (BA 40) were significantly more active in the PRE session compared to the POST session. The opposite contrast (POST vs. PRE) gave no suprathreshold clusters.

\section{Correlation between brain activity and task performance}

As we found that the left BA31 and the right BA40 significantly reduced their activation after the training but only the right BA40 was still significantly active in the POST session, we extracted the first eigenvariate of the BOLD signal in the cluster located in the right BA 40 in order to identify any possible relationship of this area with cognitive performance.

Both in the PRE and in the POST sessions a significant correlation was found between the PASAT-3 and the BOLD signal in the right BA 40 (PRE: $r=0.51, p=0.02$; POST: $r=$ $0.46, p=0.03$ ), indicating that patients with better performance had a higher activation of this brain region both before and after the rehabilitation treatment (Fig. 4). This relationship was confirmed when applying partial correlations to adjust for NBV (right BA 40 PRE: $r=0.50, \mathrm{p}=0.02$; POST: $r=0.48$, $\mathrm{p}=0.03)$.

\section{Comparison of PwMS to HS}

PwMS showed worse performance at PASAT-3 than HS both before $(t=8.28, p<0.001)$ and after $(t=3.18, p=0.003)$ the rehabilitative intervention (Fig. 5).

Concerning the fMRI activity the results from the contrast analysis to compare PVSAT-related activation between PwMS and HS revealed that PwMS significantly activated more clusters in both hemispheres in the PRE session (See Table 4). In the POST session, PwMS showed increased activation in some clusters located in the left frontoparietal regions compared to HS.

\section{Discussion}

In the present work, we showed that an adaptive training based on working memory improves cognitive performance in PwMS and impacts brain functional activation. Although both in PRE and POST sessions the patients' performance at PASAT-3 was lower than HS, it significantly increased after training. Moreover, at POST session PVSAT elicited in PwMS a brain activation map which was more similar to that one found in healthy participants compared to the PRE session. 
Table 2 Brain regions significantly activated during the PVSAT for the different groups and sessions (height threshold of $\mathrm{p}<0.05 \mathrm{FWE}-\mathrm{corrected}$, extent threshold of 30 voxels)

\begin{tabular}{|c|c|c|c|c|c|c|c|}
\hline Group & Cluster size & Voxel T & Voxel Z & MNI Coordinate: $\mathrm{x}$ y z (mm) & Laterality & Anatomical Location & Brodmann's Area \\
\hline \multirow{14}{*}{ HS } & \multirow[t]{3}{*}{577} & 13.02 & 6.31 & 122052 & Right & Cingulate Gyrus & 32 \\
\hline & & 11.64 & 6.03 & -122632 & Left & Cingulate Gyrus & 32 \\
\hline & & 9.55 & 5.54 & -141042 & Left & Cingulate Gyrus & 32 \\
\hline & \multirow[t]{3}{*}{199} & 10.33 & 5.74 & $-30-4854$ & Left & Precuneus & 7 \\
\hline & & 8.19 & 5.15 & $-46-4856$ & Left & Inferior Parietal Lobule & 40 \\
\hline & & 7.58 & 4.95 & $-48-4848$ & Left & Inferior Parietal Lobule & 40 \\
\hline & \multirow[t]{3}{*}{285} & 10.1 & 5.68 & $-42-438$ & Left & Precentral Gyrus & 6 \\
\hline & & 8.94 & 5.37 & -32042 & Left & Middle Frontal Gyrus & 6 \\
\hline & & 8.74 & 5.31 & $-42-846$ & Left & Precentral Gyrus & 6 \\
\hline & \multirow[t]{3}{*}{198} & 9 & 5.39 & $0-68-24$ & Right & Cerebellum & \\
\hline & & 8.22 & 5.16 & $14-58-28$ & Right & Cerebellum & \\
\hline & & 8.05 & 5.1 & $0-52-26$ & Right & Cerebellum & \\
\hline & \multirow[t]{2}{*}{113} & 8.52 & 5.25 & -2464 & Left & Putamen & \\
\hline & & 7.92 & 5.06 & -30166 & Left & Claustrum & \\
\hline \multirow[t]{18}{*}{ PwMS (PRE) } & \multirow[t]{3}{*}{3818} & 15.02 & 6.65 & -26058 & Left & Sub-Gyral & 6 \\
\hline & & 14.08 & 6.49 & -61048 & Left & Medial Frontal Gyrus & 32 \\
\hline & & 13.09 & 6.32 & -48822 & Left & Inferior Frontal Gyrus & 44 \\
\hline & \multirow[t]{3}{*}{1894} & 12.25 & 6.16 & $-30-4836$ & Left & Inferior Parietal Lobule & 40 \\
\hline & & 11.77 & 6.06 & $-42-5048$ & Left & Inferior Parietal Lobule & 40 \\
\hline & & 10.91 & 5.87 & $-14-7252$ & Left & Precuneus & 7 \\
\hline & \multirow[t]{2}{*}{750} & 12 & 6.11 & $28-6440$ & Right & Superior Parietal Lobule & 7 \\
\hline & & 7.54 & 4.93 & $14-6850$ & Right & Precuneus & 7 \\
\hline & 232 & 10.45 & 5.77 & $34-60-36$ & Right & Cerebellum & \\
\hline & \multirow[t]{3}{*}{642} & 10.34 & 5.74 & $48-3644$ & Right & Inferior Parietal Lobule & 40 \\
\hline & & 10.09 & 5.68 & $36-3632$ & Right & Postcentral Gyrus & 2 \\
\hline & & 9.18 & 5.44 & $40-4244$ & Right & Inferior Parietal Lobule & 40 \\
\hline & \multirow[t]{2}{*}{188} & 9.4 & 5.5 & 34220 & Right & Insula & 13 \\
\hline & & 9.34 & 5.48 & 32228 & Right & Insula & 13 \\
\hline & 48 & 8.91 & 5.36 & $-30-7826$ & Left & Superior Occipital Gyrus & 39 \\
\hline & 117 & 8.87 & 5.35 & 444618 & Right & Middle Frontal Gyrus & 10 \\
\hline & 51 & 7.7 & 4.99 & $-34-60-30$ & Left & Cerebellum & \\
\hline & 32 & 7.33 & 4.86 & $10-72-34$ & Right & Cerebellum & \\
\hline \multirow[t]{13}{*}{ PwMS (POST) } & \multirow[t]{3}{*}{752} & 11.44 & 5.99 & $-26-6440$ & Left & Superior Parietal Lobule & 7 \\
\hline & & 10.69 & 5.82 & $-16-6246$ & Left & Precuneus & 7 \\
\hline & & 9.51 & 5.53 & $-10-6650$ & Left & Precuneus & 7 \\
\hline & 65 & 9.92 & 5.64 & $26-64-52$ & Right & Cerebellum & \\
\hline & 42 & 9.61 & 5.55 & $-42-1036$ & Left & Precentral Gyrus & 6 \\
\hline & 63 & 9.41 & 5.5 & $34-58-34$ & Right & Cerebellum & \\
\hline & \multirow[t]{2}{*}{186} & 8.53 & 5.25 & -50822 & Left & Inferior Frontal Gyrus & 44 \\
\hline & & 8.19 & 5.15 & -40426 & Left & Inferior Frontal Gyrus & 9 \\
\hline & 33 & 8.35 & 5.2 & $42-3832$ & Right & Inferior Parietal Lobule & 40 \\
\hline & \multirow[t]{2}{*}{118} & 8.24 & 5.16 & -6852 & Left & Medial Frontal Gyrus & 32 \\
\hline & & 7.87 & 5.04 & -2662 & Left & Superior Frontal Gyrus & 6 \\
\hline & \multirow[t]{2}{*}{59} & 8.07 & 5.11 & -482226 & Left & Middle Frontal Gyrus & 46 \\
\hline & & 7.96 & 5.07 & -463624 & Left & Middle Frontal Gyrus & 46 \\
\hline
\end{tabular}

The proposed rehabilitative intervention was very well received by all PwMS, in line with a previous study investigating the usability of COGNI-TRAcK (Tacchino et al. 2015). This result could be related to the advantages of a system allowing an at-home treatment, which avoids frequent travels to the rehabilitation center.

The improvement in cognitive performance observed following the proposed adaptive training based on WM is in line with other studies investigating the effects of cognitive rehabilitation in MS (Amato et al. 2014; Chiaravalloti et al. 2013; Filippi et al. 2012; Mattioli et al. 2010; Pedullà et al. 2016). The adaptive algorithm allowed the patients to train at their maximal working load throughout the whole amount of the intervention, as shown by the increase of the difficulty levels in spite of a constant accuracy percentage. It is worth noting that in the $N$-back tasks reaction time decreased from the first to the last session of the training although the difficulty of the exercises increased. This is an important result since previous research showed that PwMS responded significantly slower than controls on reaction time tests and that the difference between the performances of the two groups progressively increased as the tests became more difficult, i.e. as processing demands increased (Reicker et al. 2007). In our study participants improved their speed of processing ability similarly to a 


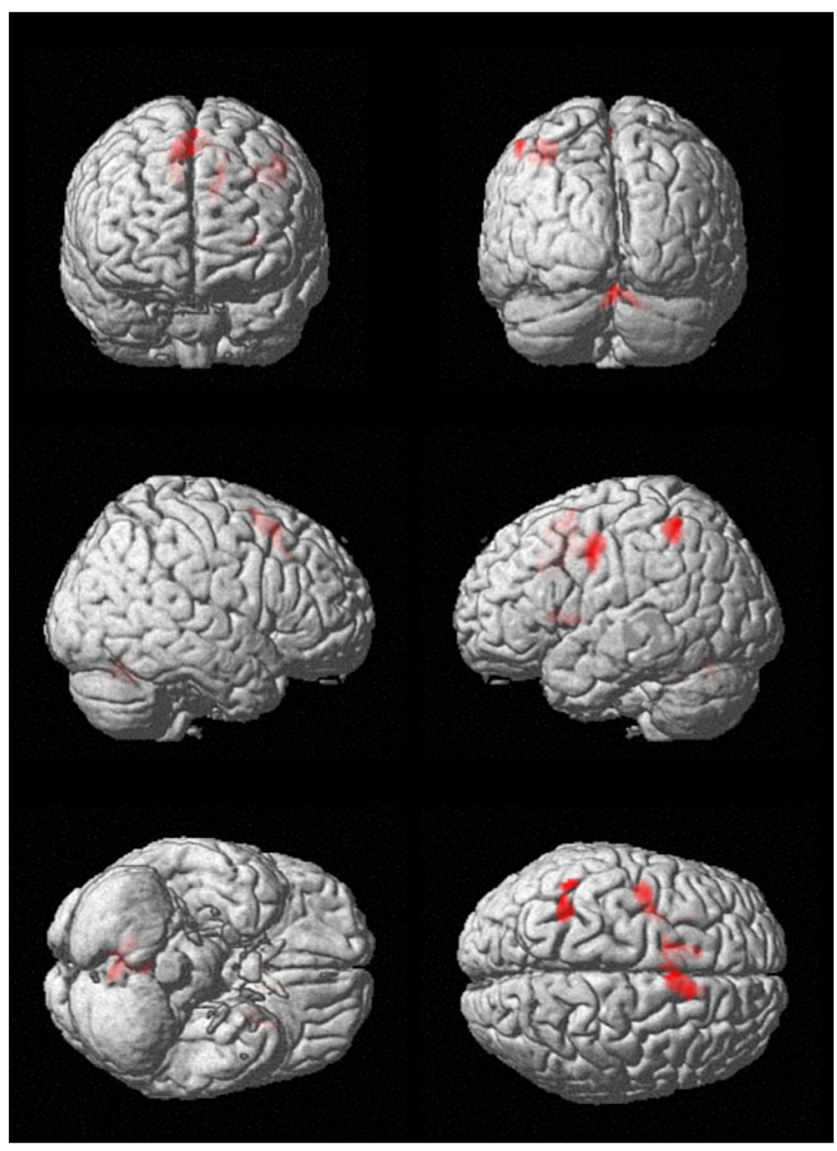

Fig. 2 Group activation map of healthy subjects during the PVSAT (see Table 2 for details). Significant blobs are displayed on a rendering surface (height threshold $p<0.05$ FWE-corrected; extent threshold $\mathrm{k}=30$ voxels)

sample of PwMS who underwent a specific computer based home training of divided attention that aimed to improve this cognitive function (Pusswald et al. 2014). Thus, we may suggest that the proposed adaptive training based on WM has a positive effect on trained (e.g., information processing speed, sustained attention and visual memory) and non-trained (e.g., alertness, divided attention, verbal memory and fluency) cognitive domains, corroborating previous evidence supporting a mediating/intervening role of WM in other cognitive functions in PwMS (Berrigan et al. 2013).

Importantly, intervention-associated changes were not only statistically significant, but also meaningful in terms of magnitude of the difference, as shown by the effect size analysis. Moreover, improvements in SDMT and PASAT-3 were widely above the percentage accepted in the literature as minimally clinically relevant difference (reported as 10\% for SDMT (Benedict et al. 2017) and 20\% for PASAT-3 (Hoogervorst et al. 2004)).

The lack of a control group of PwMS does not allow disentangling between training effects and other unspecific effects such as time or the repetition of assessment. However, in order to minimize form-specific practice effects, equivalent forms were used and participants were randomly assigned to receive Form A or B in the PRE session and then switched in the POST session. Thus, we can suggest that the improvement in cognitive performance in PwMS measured in this study was a direct effect of the treatment.

In parallel to cognitive performance improvement, we found a significant reduction of cortical activity after COGNI-TRAcK treatment. In particular, contrast analysis identified the left cingulate gyrus and the right inferior parietal lobule (BA40) as the areas where activity significantly reduced after the intervention.

Conversely, other authors have shown increased activation of existing networks underlying trained functions in PwMS following cognitive rehabilitation (Chiaravalloti et al. 2012; Ernst et al. 2012). Neuroplasticity of cognitive functions in PwMS can occur thanks to different neural processes concerning homologous region adaptation, local activation expansion, and extra-region recruitment towards the maintenance of cognitive functioning (Chiaravalloti et al. 2015). In our study, we found abnormal brain activation during PVSAT compared to healthy subjects before treatment, with a pattern of activation including several bilateral activation clusters. In cross-sectional studies it is generally not easy to differentiate the adaptive components of plasticity due to functional architecture response to tissue damage from maladaptive changes that negatively impact cognitive performance. Here, however, thanks to our longitudinal design we observed that improvement in cognitive performance over time was associated with a size reduction of the activation pattern thus indirectly suggesting a recovery from a possible condition of maladaptive neuroplasticity. Maladaptive neuroplasticity may come at the cost of other cognitive functions. Therefore, effective cognitive rehabilitation programs should be aimed at inducing adaptive neuroplasticity, "normalizing" brain function and behavioral output. Following our cognitive rehabilitation program, PwMS showed reduced task-related activation with clusters mainly located in the right cerebellum and in the left-brain hemisphere; the only significant cluster in the right hemisphere was located in the inferior parietal lobule (BA 40).

These findings may imply that WM training can improve the cognitive performance and reduce the abnormal activation of cognitively impaired PwMS, partially maintaining or even restoring the brain cognitive function of PwMS and restricting the neural recruitment to the only brain areas that are responsible for the maintenance of function (greater "brain efficiency"). Especially, we could suggest that adaptive training based on WM could be extremely useful because of its ability to tune step-by-step performance. Indeed, lowering the level of tasks difficulty in the case of unsuccessful performance might reduce the stress encountered by the individual. On the other hand, the tailored difficulty increase consequential to patient improvement might physiologically activate existing 


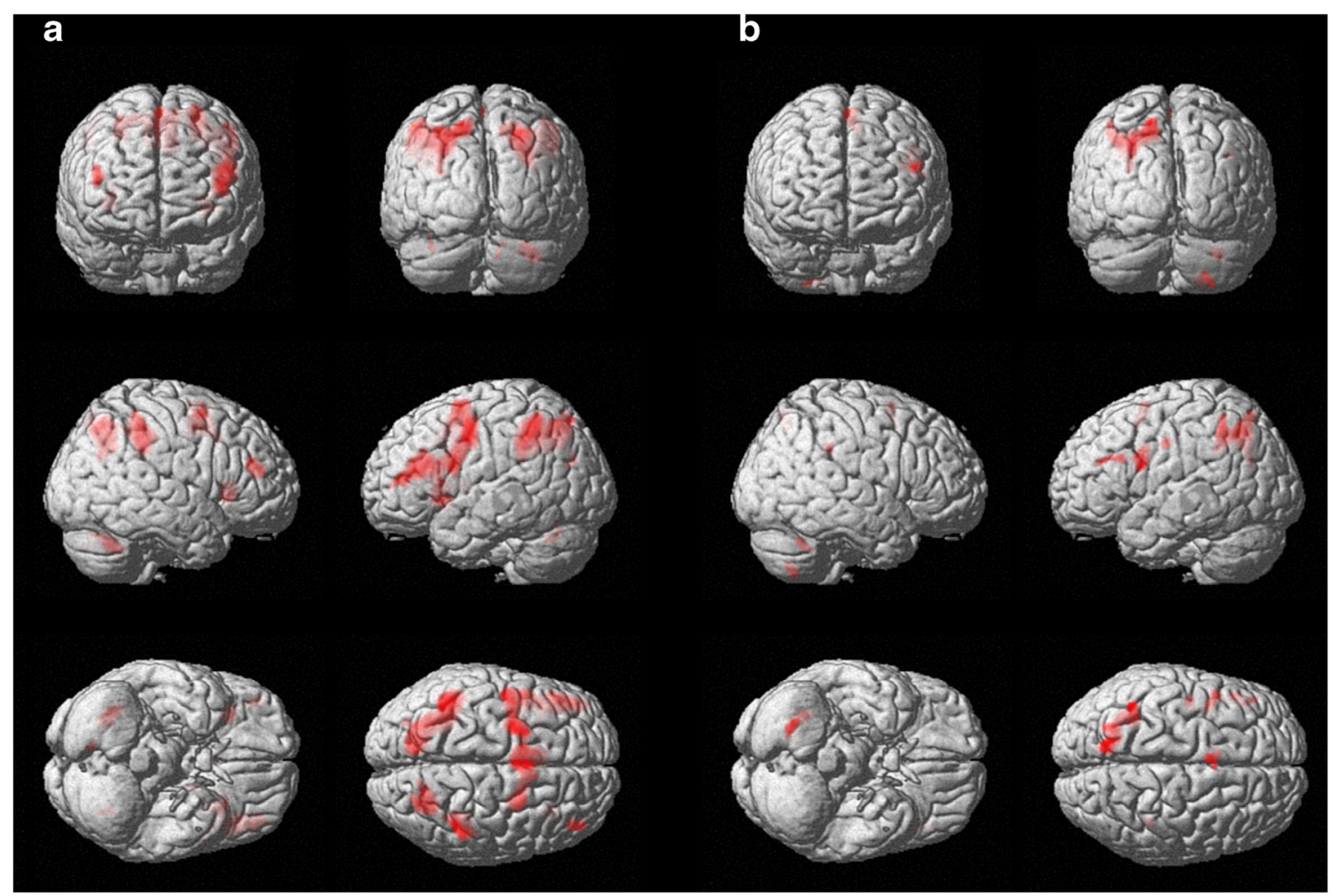

Fig. 3 Group activation blobs displayed on a rendering surface for the PwMS group (height threshold $\mathrm{p}<0.05$ FWE-corrected; extent threshold $\mathrm{k}=30$ voxels) in the two experimental sessions: a before the rehabilitative treatment, $\mathbf{b}$ after the rehabilitative treatment (see Table 2 for details)

networks for cognitive functioning stimulating a more accurate compensatory adaptive process (Voytek et al. 2010).

The right BA40 could represent a basic compensatory area whose activation may help coping for brain damage in PwMS in order to sustain attentional tasks. This hypothesis is strongly supported by the positive partial correlation between the PASAT-3 scores and the right BA40 BOLD signal when controlling for brain atrophy (here expressed as NBV), which was significant both at PRE and at POST sessions.

The right inferior parietal lobule has been shown to be involved both in mathematical processing (Chochon et al. 1999) and attentional resources allocation (Singh-Curry and Husain 2009), which represent two of the key components underlying PVSAT performance. In line with these findings, a crucial role of the inferior parietal lobule in working memory and sustained attention tasks, such as the PVSAT, was observed in a recent meta-analysis conducted on both healthy controls and PwMS (Kollndorfer et al. 2013). The inferior parietal lobule has been demonstrated to be more active during the 2-back task in PwMS with preserved cognition compared to those with cognitive deficits (Rocca et al. 2010) and to present increased functional connectivity with the default mode network after cognitive rehabilitation (Bonavita et al. 2015). Moreover, a resting-state investigation involving PwMS showed increased functional connectivity of the right inferior parietal lobule after cognitive treatment (Parisi et al. 2014). The functional connectivity between the anterior cingulate cortex and the right BA40 was also found to correlate with improvement of PASAT performance, suggesting the occurrence of compensatory mechanisms after cognitive training.

In particular, the right inferior parietal lobule could have a role in cognitive reserve (CR), referred to as the brain active

Table 3 Brain regions showing significantly different activation during the PVSAT in the PwMS group between the two sessions (height threshold of $p<0.001$ uncorrected, extent threshold of 30 voxels)

Contrast Cluster Size Voxel T Voxel Z MNI Coordinate: x y z (mm) Laterality Anatomical Location Brodmann's

Area

\begin{tabular}{llllllll}
\hline PwMS (PRE) - PwMS (POST) & 41 & 4.8 & 3.76 & $-12-2638$ & Left & Cingulate Gyrus & 31 \\
& 36 & 4.14 & 3.4 & $48-3652$ & Right & Postcentral Gyrus & 40 \\
& & 3.94 & 3.27 & $56-3448$ & Right & Inferior Parietal Lobule & 40
\end{tabular}

PwMS (POST) - PwMS (PRE) No suprathreshold clusters 
Fig. 4 Linear relationship between PASAT-3 and the first eigenvariate of the BOLD signal in the right $\mathrm{BA} 40$ before (a) and after (b) the cognitive rehabilitation treatment

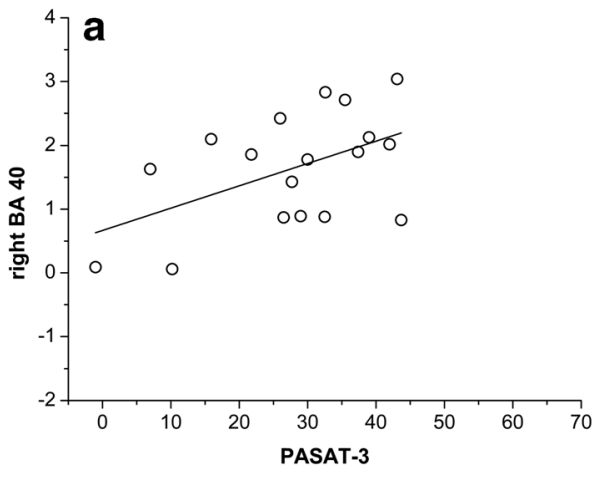

attempt to cope with brain damage using pre-existing cognitive processes or enlisting compensatory strategies (Stern 2002). Indeed, using both fMRI and PET imaging, the inferior parietal lobule has been shown in healthy subjects to be included in a frontoparietal network modulated by CR (Stern et al. 2003, 2005). The CR model could explain the lack of a direct relationship between the extent of brain pathology and the clinical manifestation of cognitive impairment in MS (Benedict et al. 2010; Sumowski et al. 2009).

In this scenario, there is increasing interest for applications able to build cognitive reserve and thus to preserve (or even ameliorate) cognitive functions during the MS course (see Sandroff et al. 2016 for a review). In fact, there is emerging evidence that CR may not be limited to premorbid factors (i.e., genetics, education and early-life behavior) that are not highly amenable to change but also could be impacted by training programs.

As previously suggested by Sandry and Sumowski, 2014, WM seems to act as a "memory buffer" mediating cognitive performance and individual aspects which can relate either to premorbid characteristics (e.g., genetics, education and neural

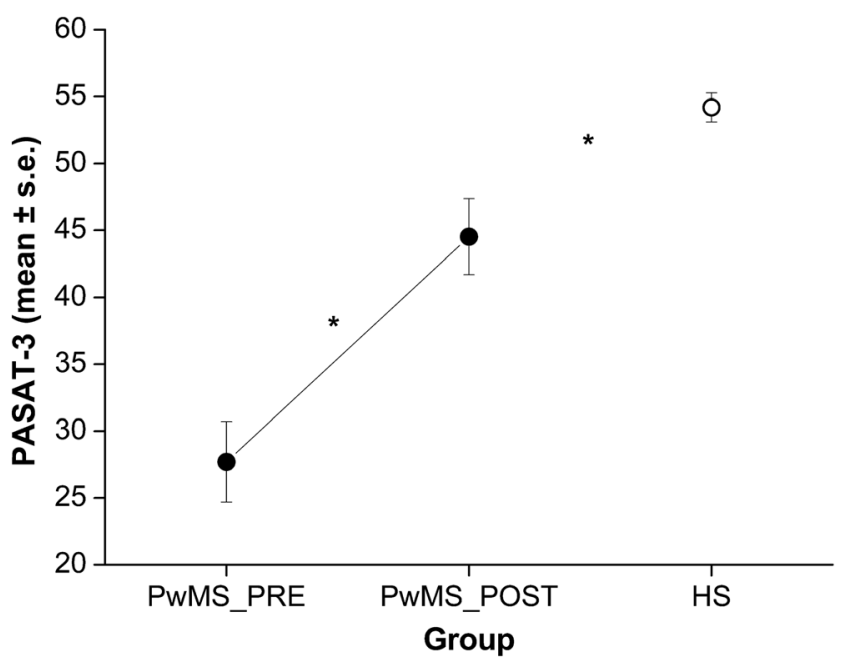

Fig. 5 PASAT-3 corrected scores of the PwMS group, before (PRE) and after (POST) the rehabilitative intervention, and of the group of healthy subjects (HS). * indicate $p<0.05$ (PwMS_POST vs. PwMS_PRE and PwMS_post vs. HS) functioning) or to disease-mediated factors, such as brain damage and dysfunctions (Fig. 6). As WM capacity increased following a specific, intensive and personalized training, compensatory areas that spontaneously activated in the attempt to maintain function reduced their activity. At the same time, overall cognitive performance was at least partly restored, as shown by the increased scores obtained by the patients at the neuropsychological battery after the intervention.

Limitations of the present study include the small sample size (which probably prevented us from obtaining significant results at a corrected threshold in the contrast analysis) and the lack of a control group of patients receiving a non-adaptive intervention. Also, the healthy group underwent only one assessment session. Moreover, while we did take into account in our analyses the differences in brain volume (a major determinant of cognitive performance), we did not correct for clinical variables nor treatments or white matter lesions, given the proof-of-concept nature of this work. Therefore, these results should be interpreted with caution and not generalized to the all population of PwMS.

A limitation regarding data interpretation is represented by the use of the PVSAT paradigm which is impacted by confounding factors such as anxiety and it is not a pure measure of a single cognitive function. Especially, PVSAT paradigm probes different constructs such as processing speed, attention and working memory, which are mediated by only partly overlapping neural networks (Olivers et al. 2011).

In conclusion, we suggest that an adaptive training based on WM (in this study administered by means of COGNITRAcK) can enhance the brain ability to cope with tissue damage to maintain or even partly restore cognitive functioning. In addition, this study showed that the right BA40 seems to be a "core" of functional reserve for cognitive performance in MS, since it was still active after the cognitive rehabilitation treatment (differently from other areas, such as the left BA 31) and its BOLD signal significantly correlated with cognitive performance. These findings open new perspectives on the role of the right BA40 in cognitive functioning in people with neurological disorders. In particular, further longitudinal studies should aim to monitor this area along the course of the disease or during longer rehabilitative treatments, in order to evaluate the efficacy of interventions also at a neural level. 
Table 4 Brain regions showing significantly different activation during the PVSAT between groups (height threshold of $\mathrm{p}<0.001$ uncorrected, extent threshold of 30 voxels)

\begin{tabular}{|c|c|c|c|c|c|c|c|}
\hline Contrast & Cluster Size & Voxel T & Voxel Z & MNI Coordinate: x y z (mm) & Laterality & Anatomical Location & Brodmann's Area \\
\hline \multirow{22}{*}{ PwMS (PRE) - HS } & \multirow[t]{3}{*}{423} & 5.70 & 4.74 & -81236 & Left & Cingulate Gyrus & 24 \\
\hline & & 3.97 & 3.57 & -12454 & Left & Medial Frontal Gyrus & 6 \\
\hline & & 3.65 & 3.33 & -201652 & Left & Superior Frontal Gyrus & 8 \\
\hline & \multirow[t]{3}{*}{696} & 5.69 & 4.73 & -404212 & Left & Middle Frontal Gyrus & 10 \\
\hline & & 5.02 & 4.31 & -482816 & Left & Inferior Frontal Gyrus & 46 \\
\hline & & 4.74 & 4.12 & -442018 & Left & Inferior Frontal Gyrus & 45 \\
\hline & \multirow[t]{2}{*}{871} & 5.52 & 4.63 & $-42-5250$ & Left & Inferior Parietal Lobule & 40 \\
\hline & & 4.16 & 3.71 & $-60-4038$ & Left & Inferior Parietal Lobule & 40 \\
\hline & \multirow[t]{3}{*}{532} & 5.46 & 4.60 & $-32-240$ & Left & Precentral Gyrus & 6 \\
\hline & & 4.76 & 4.14 & $-40-640$ & Left & Precentral Gyrus & 6 \\
\hline & & 4.20 & 3.74 & $-34-224$ & Left & Insula & 13 \\
\hline & 143 & 4.79 & 4.16 & 30244 & Right & Insula & 13 \\
\hline & \multirow[t]{2}{*}{275} & 4.52 & 3.97 & $-22-7644$ & Left & Superior Parietal Lobule & 7 \\
\hline & & 4.04 & 3.63 & $-12-7650$ & Left & Precuneus & 7 \\
\hline & \multirow[t]{3}{*}{326} & 4.40 & 3.88 & $56-2834$ & Right & Inferior Parietal Lobule & 40 \\
\hline & & 4.20 & 3.74 & $42-3428$ & Right & Inferior Parietal Lobule & 40 \\
\hline & & 3.59 & 3.28 & $64-2638$ & Right & Postcentral Gyrus & 2 \\
\hline & 67 & 4.38 & 3.87 & $28-6844$ & Right & Superior Parietal Lobule & 7 \\
\hline & 43 & 4.29 & 3.81 & -28162 & Left & Claustrum & \\
\hline & 38 & 4.01 & 3.60 & 36622 & Right & Insula & 13 \\
\hline & \multirow[t]{2}{*}{72} & 3.91 & 3.53 & $-10-6038$ & Left & Precuneus & 7 \\
\hline & & 3.65 & 3.33 & $-4-6836$ & Left & Precuneus & 7 \\
\hline HS - PwMS (PRE) & \multicolumn{7}{|c|}{ No suprathreshold clusters } \\
\hline \multirow[t]{5}{*}{ PwMS (POST) - HS } & \multirow[t]{2}{*}{122} & 4.48 & 3.94 & -364212 & Left & Middle Frontal Gyrus & 10 \\
\hline & & 4.01 & 3.6 & -443814 & Left & Inferior Frontal Gyrus & 46 \\
\hline & 37 & 4.13 & 3.69 & -121432 & Left & Cingulate Gyrus & 24 \\
\hline & 45 & 3.95 & 3.56 & $-8-6238$ & Left & Precuneus & 7 \\
\hline & 46 & 3.92 & 3.53 & $-32-236$ & Left & Precentral Gyrus & 6 \\
\hline HS - PwMS (POST) & 60 & 4.02 & 3.61 & $40-246$ & Right & Superior Temporal Gyrus & 13 \\
\hline
\end{tabular}
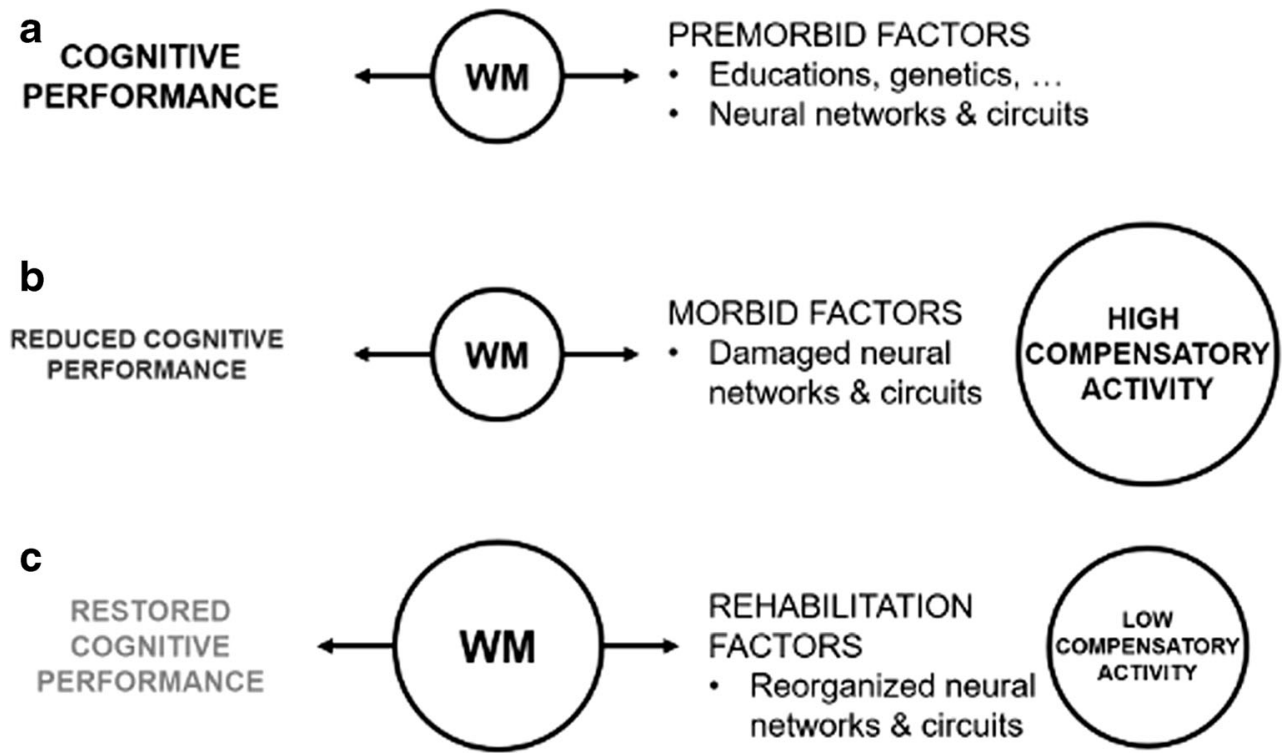

REHABILITATION

\section{FACTORS}

- Reorganized neural networks \& circuits

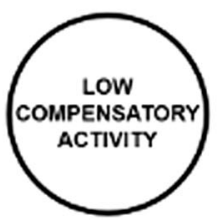

Fig. 6 Theoretical framework of cognitive reserve functioning in MS. WM was shown to be a mechanism linking cognitive performance (in particular in a task of long-term memory) and individual factors (Sandry and Sumowski 2014) (a). In this and previous studies (Bonzano et al. 2009) we demonstrated that in PwMS brain damage leads to increased widespread neural activity, in spite of a reduced cognitive performance (b). However, an effective cognitive rehabilitation treatment can increase WM capacity, reduce compensatory activity and restore, at least in part, cognitive performance (c) 
Acknowledgements This work was supported by the Italian Multiple Sclerosis Foundation - FISM (project n. 2011/R/8) and was jointly carried out by the formal collaboration between the Department of Experimental Medicine of the University of Genoa and FISM Scientific Research Area, titled "Neural plasticity in the rehabilitation of the sensorimotor system".

Open Access This article is distributed under the terms of the Creative Commons Attribution 4.0 International License (http:// creativecommons.org/licenses/by/4.0/), which permits unrestricted use, distribution, and reproduction in any medium, provided you give appropriate credit to the original author(s) and the source, provide a link to the Creative Commons license, and indicate if changes were made.

\section{References}

Altman, D. G. (2009). Missing outcomes in randomized trials: Addressing the dilemma. Open Medicine.

Amann, M., Dö, L. S., Penner, I.-K., Hirsch, J. G., Raselli, C., Calabrese, P., et al. (2011). Altered functional adaptation to attention and working memory tasks with increasing complexity in relapsing-remitting multiple sclerosis patients. Human Brain Mapping, 32, 1704-1719.

Amato, M., Portaccio, E., Goretti, B., Zipoli, V., Ricchiuti, L., De Caro, M., et al. (2006a). The Rao's brief repeatable battery and Stroop test: Normative values with age, education and gender corrections in an Italian population. Multiple Sclerosis, 12, 787-793.

Amato, M., Zipoli, V., \& Portaccio, E. (2006b). Multiple sclerosis-related cognitive changes: A review of cross-sectional and longitudinal studies. Journal of the Neurological Sciences, 245(1-2), 41-46.

Amato, M. P., Portaccio, E., Goretti, B., Zipoli, V., Iudice, A., Pina, D. D., Malentacchi, G., Sabatini, S., Annunziata, P., Falcini, M., Mazzoni, M., Mortilla, M., Fonda, C., de Stefano, N., \& TuSCIMS Study Group. (2010). Relevance of cognitive deterioration in early relapsing-remitting MS: A 3-year follow-up study. Multiple Sclerosis, 16(12), 1474-1482.

Amato, M. P., Goretti, B., Viterbo, R. G., Portaccio, E., Niccolai, C., Hakiki, B., Iaffaldano, P., \& Trojano, M. (2014). Computerassisted rehabilitation of attention in patients with multiple sclerosis: Results of a randomized, double-blind trial. Multiple sclerosis (Houndmills, Basingstoke, England), 20, 91-98.

Audoin, B., Ibarrola, D., Duong, A., V, M., Pelletier, J., Confort-Gouny, S., Malikova, I., et al. (2005a). Functional MRI study of PASAT in normal subjects. Magnetic Resonance Materials in Physics, Biology and Medicine, 18(2), 96-102.

Audoin, B., Van, A., Duong, M., Ranjeva, J. P., Ibarrola, D., Malikova, I., Confort-Gouny, S., et al. (2005b). Magnetic resonance study of the influence of tissue damage and cortical reorganization on PASAT performance at the earliest stage of multiple sclerosis. Human Brain Mapping, 24, 216-228.

Benedict, R., Morrow, S., Weinstock Guttman, B., Cookfair, D., \& Schretlen, D. (2010). Cognitive reserve moderates decline in information processing speed in multiple sclerosis patients. Journal of the International Neuropsychological Society : JINS, 16(5), 829-835.

Benedict, R. H., DeLuca, J., Phillips, G., LaRocca, N., Hudson, L. D., Rudick, R., \& Consortium, M. S. O. A. (2017). Validity of the symbol digit modalities test as a cognition performance outcome measure for multiple sclerosis. Multiple Sclerosis Journal, 23(5), 721-733.

Berrigan, L. I., Lefevre, J. A., Rees, L. M., Berard, J., Freedman, M. S., \& Walker, L. A. S. (2013). Cognition in early relapsing-remitting multiple sclerosis: Consequences may be relative to working memory. Journal of the International Neuropsychological Society, 19(8), 938-949.

Bonavita, S., Sacco, R., Della Corte, M., Esposito, S., Sparaco, M., D'ambrosio, A., et al. (2015). Computer-aided cognitive rehabilitation improves cognitive performances and induces brain functional connectivity changes in relapsing remitting multiple sclerosis patients: An exploratory study. Journal of Neurology, 262, 91-100.

Bonzano, L., Pardini, M., Mancardi, G. L., Pizzorno, M., \& Roccatagliata, L. (2009). Structural connectivity influences brain activation during PVSAT in multiple sclerosis. NeuroImage, 44(1), 9-15.

Cader, S., Cifelli, A., Abu-Omar, Y., Palace, J., \& Matthews, P. M. (2006). Reduced brain functional reserve and altered functional connectivity in patients with multiple sclerosis. Brain, 129(2), 527-537.

Chiaravalloti, N. D., \& DeLuca, J. (2008). Cognitive impairment in multiple sclerosis. The Lancet Neurology, 7(12), 1139-1151.

Chiaravalloti, N., Hillary, F., Ricker, J., Christodoulou, C., Kalnin, A., Liu, W.-C., et al. (2005). Cerebral activation patterns during working memory performance in multiple sclerosis using FMRI. Journal of Clinical and Experimental Neuropsychology, 27(1), 33-54.

Chiaravalloti, N. D., Wylie, G., Leavitt, V., \& DeLuca, J. (2012). Increased cerebral activation after behavioral treatment for memory deficits in MS. Journal of Neurology, 259(7), 1337-1346.

Chiaravalloti, N. D., Stojanovic-Radic, J., \& DeLuca, J. (2013). The role of speed versus working memory in predicting learning new information in multiple sclerosis. Journal of Clinical and Experimental Neuropsychology, 35(2), 180-191.

Chiaravalloti, N. D., Genova, H. M., \& DeLuca, J. (2015). Cognitive rehabilitation in multiple sclerosis: The role of plasticity. Frontiers in Neurology, 6, 67.

Chochon, F., Cohen, L., van de Moortele, P., \& Dehaene, S. (1999). Differential contributions of the left and right inferior parietal lobules to number processing. Journal of Cognitive Neuroscience, 11(6), 617-630.

Cohen, J. (1988). Statistical power analysis for the behavioral sciences. Statistical Power Analysis for the Behavioral Sciences (Elsevier., Vol. 2nd).

Dackovic, J., Pekmezovic, T., Mesaros, S., Dujmovic, I., Stojsavljevic, N., Martinovic, V., \& Drulovic, J. (2016). The Rao's brief repeatable battery in the study of cognition in different multiple sclerosis phenotypes: Application of normative data in a Serbian population. Neurological Sciences, 37(9), 1475-1481.

DeLuca, G. C., Yates, R. L., Beale, H., \& Morrow, S. A. (2015). Cognitive impairment in multiple sclerosis: Clinical, radiologic and pathologic insights. Brain Pathology, 25(1), 79-98.

Ernst, A., Botzung, A., Gounot, D., Sellal, F., Blanc, F., de Seze, J., \& Manning, L. (2012). Induced brain plasticity after a facilitation Programme for autobiographical memory in multiple sclerosis: A preliminary study. Multiple Sclerosis International, 2012, 1-12.

Filippi, M., Riccitelli, G., Mattioli, F., Capra, R., Stampatori, C., Pagani, E., Valsasina, P., Copetti, M., Falini, A., Comi, G., \& Rocca, M. A. (2012). Multiple sclerosis: Effects of cognitive rehabilitation on structural and functional MR imaging measures-an explorative study. Radiology, 262(3), 932-940.

Flachenecker, P., Kümpfel, T., Kallmann, B., Gottschalk, M., Grauer, O., Rieckmann, P., Trenkwalder, C., \& Toyka, K. V. (2002). Fatigue in multiple sclerosis: A comparison of different rating scales and correlation to clinical parameters. Multiple Sclerosis, 8(February), 523-526.

Forn, C., Barros-Loscertales, A., Escudero, J., Belloch, V., Campos, S., Parcet, M. A., \& Ávila, C. (2006). Cortical reorganization during PASAT task in MS patients with preserved working memory functions. NeuroImage, 31, 686-691.

Forn, C., Barros-Loscertales, A., Escudero, J., Benlloch, V., Campos, S., Parcet, M. A., \& Ávila, C. (2007). Compensatory activations in patients with multiple sclerosis during preserved performance on the auditory n-back task. Human Brain Mapping, 28(5), 424-430.

Friston, K. J., Holmes, A. P., Poline, J. B., Grasby, P. J., Williams, S. C., Frackowiak, R. S., \& Turner, R. (1995). Analysis of fMRI timeseries revisited. NeuroImage, 2(1), 45-53.

Goretti, B., Patti, F., Cilia, S., Mattioli, F., Stampatori, C., Scarpazza, C., Amato, M. P., \& Portaccio, E. (2014). The Rao's brief repeatable battery version B: Normative values with age, education and gender 
corrections in an Italian population. Neurological Sciences, 35(1), 79-82.

Hillary, F. G., Chiaravalloti, N. D., Ricker, J. H., Steffener, J., Bly, B. M., Lange, G., Liu, W. C., Kalnin, A. J., \& DeLuca, J. (2003). An investigation of working memory rehearsal in multiple sclerosis using fMRI. Journal of Clinical and Experimental Neuropsychology, 25(7), 965-978.

Hoogervorst, E., Kalkers, N., Cutter, G., Uitdehaag, B., \& Polman, C. (2004). The patient's perception of a (reliable) change in the multiple sclerosis functional composite. Multiple Sclerosis, 10, 55-60.

Kawashima, R., Okita, K., Yamazaki, R., Tajima, N., Yoshida, H., Taira, M., Iwata, K., Sasaki, T., Maeyama, K., Usui, N., \& Sugimoto, K. (2005). Reading aloud and arithmetic calculation improve frontal function of people with dementia. The Journals of Gerontology. Series A, Biological Sciences and Medical Sciences, 60(3), 380 384.

Kollndorfer, K., Krajnik, J., Woitek, R., Freiherr, J., Prayer, D., \& Schöpf, V. (2013). Altered likelihood of brain activation in attention and working memory networks in patients with multiple sclerosis: An ALE meta-analysis. Neuroscience and Biobehavioral Reviews, 37(10), 2699-2708.

Kurtzke, J. F. (1983). Rating neurologic impairment in multiple sclerosis: An expanded disability status scale (EDSS). Neurology, 33, 1444 1452

Mainero, C., Caramia, F., Pozzilli, C., Pisani, A., Pestalozza, I., Borriello, G., Bozzao, L., \& Pantano, P. (2004). fMRI evidence of brain reorganization during attention and memory tasks in multiple sclerosis. NeuroImage, 21(3), 858-867.

Mattioli, F., Stampatori, C., Zanotti, D., Parrinello, G., \& Capra, R. (2010). Efficacy and specificity of intensive cognitive rehabilitation of attention and executive functions in multiple sclerosis. Journal of the Neurological Sciences, 288, 101-105.

Nagels, G., Geentjens, L., Kos, D., Vleugels, L., D’hooghe, M. B., Van Asch, P., et al. (2005). Paced visual serial addition test in multiple sclerosis. Clinical Neurology and Neurosurgery, 107(3), 218-222.

Olesen, P. J., Westerberg, H., \& Klingberg, T. (2004). Increased prefrontal and parietal activity after training of working memory. Nature Neuroscience, 7(1), 75-79.

Olivers, C. N. L., Peters, J., Houtkamp, R., \& Roelfsema, P. R. (2011). Different states in visual working memory: When it guides attention and when it does not. Trends in Cognitive Sciences, 15(7), 327-334.

Pantano, P., Mainero, C., \& Caramia, F. (2006). Functional brain reorganization in multiple sclerosis: Evidence from fMRI studies. Journal of neuroimaging : official journal of the American Society of Neuroimaging, 16(2), 104-114.

Parisi, L., Rocca, M. A., Valsasina, P., Panicari, L., Mattioli, F., \& Filippi, M. (2014). Cognitive rehabilitation correlates with the functional connectivity of the anterior cingulate cortex in patients with multiple sclerosis. Brain Imaging and Behavior, 8(3), 387-393.

Pedullà, L., Brichetto, G., Tacchino, A., Vassallo, C., Zaratin, P., Battaglia, M. A., Bonzano, L., \& Bove, M. (2016). Adaptive vs. non-adaptive cognitive training by means of a personalized app: A randomized trial in people with multiple sclerosis. Journal of Neuroengineering and Rehabilitation, 13(1), 88.

Pusswald, G., Mildner, C., Zebenholzer, K., Auff, E., \& Lehrner, J. (2014). A neuropsychological rehabilitation program for patients with multiple sclerosis based on the model of the ICF. NeuroRehabilitation, 35(3), 519-527.

Rao, S. M., \& The Cognitive Function Study Group of the National Multiple Sclerosis Association. (1990). A manual for the brief, repeatable battery of neuropsychological tests in multiple sclerosis. Milwaukee.

Reicker, L. I., Tombaugh, T. N., Walker, L., \& Freedman, M. S. (2007). Reaction time: An alternative method for assessing the effects of multiple sclerosis on information processing speed. Archives of Clinical Neuropsychology, 22(5), 655-664.
Rocca, M. A., Riccitelli, G., Rodegher, M., Ceccarelli, A., Falini, A., Falautano, M., Meani, A., Comi, G., \& Filippi, M. (2010). Functional MR imaging correlates of neuropsychological impairment in primary-progressive multiple sclerosis. American Journal of Neuroradiology, 31(7), 1240-1246.

Sandroff, B. M., Schwartz, C. E., \& DeLuca, J. (2016). Measurement and maintenance of reserve in multiple sclerosis. Journal of Neurology, 263(11), 2158-2169.

Sandry, J., \& Sumowski, J. F. (2014). Working memory mediates the relationship between intellectual enrichment and long-term memory in multiple sclerosis: An exploratory analysis of cognitive reserve. Journal of the International Neuropsychological Society : JINS, 20(8), 1-5.

Sawilowsky, S. S. (2009). New effect size rules of thumb. Journal of Modern Applied Statistical Methods, 8(2), 597-599.

Singh-Curry, V., \& Husain, M. (2009). The functional role of the inferior parietal lobe in the dorsal and ventral stream dichotomy. Neuropsychologia, 47(6), 1434-1448.

Smith, S. M., Zhang, Y., Jenkinson, M., Chen, J., Matthews, P. M., Federico, A., \& De Stefano, N. (2002). Accurate, robust, and automated longitudinal and cross-sectional brain change analysis. NeuroImage, 17(1), 479-489.

Staffen, W., Mair, A., Zauner, H., Unterrainer, J., Niederhofer, H., Kutzelnigg, A., Ritter, S., Golaszewski, S., Iglseder, B., \& Ladurner, G. (2002). Cognitive function and fMRI in patients with multiple sclerosis: Evidence for compensatory cortical activation during an attention task. Brain : a journal of neurology, 125(Pt 6), $1275-1282$.

Stern, Y. (2002). What is cognitive reserve? Theory and research application of the reserve concept. Journal of the International Neuropsychological Society : JINS, 8(3), 448-460.

Stern, Y., Zarahn, E., Hilton, H. J., Flynn, J., DeLaPaz, R., \& Rakitin, B. (2003). Exploring the neural basis of cognitive reserve. Journal of Clinical and Experimental Neuropsychology, 25(April 2015), 691701.

Stern, Y., Habeck, C., Moeller, J., Scarmeas, N., Anderson, K. E., Hilton, H. J., Flynn, J., Sackeim, H., \& van Heertum, R. (2005). Brain networks associated with cognitive reserve in healthy young and old adults. Cerebral cortex (New York, N.Y. : 1991), 15(4), 394-402.

Sumowski, J. F., Chiaravalloti, N., \& DeLuca, J. (2009). Cognitive reserve protects against cognitive dysfunction in multiple sclerosis. Journal of Clinical and Experimental Neuropsychology, 31(8), 913-926.

Sweet, L. H., Rao, S. M., Primeau, M., Durgerian, S., \& Cohen, R. A. (2006). Functional magnetic resonance imaging response to increased verbal working memory demands among patients with multiple sclerosis. Human Brain Mapping, 27(1), 28-36.

Tacchino, A., Pedullà, L., Bonzano, L., Vassallo, C., Battaglia, M. A., Mancardi, G., Bove, M., \& Brichetto, G. (2015). A new app for athome cognitive training: Description and pilot testing on patients with multiple sclerosis. JMIR mHealth and uHealth, 3, e85.

Takeuchi, H., Sekiguchi, A., Taki, Y., Yokoyama, S., Yomogida, Y., Komuro, N., Yamanouchi, T., Suzuki, S., \& Kawashima, R. (2010). Training of working memory impacts structural connectivity. The Journal of neuroscience : the official journal of the Society for Neuroscience, 30, 3297-3303.

Uchida, S., \& Kawashima, R. (2008). Reading and solving arithmetic problems improves cognitive functions of normal aged people: A randomized controlled study. Age, 30, 21-29.

Van Schependom, J., D'hooghe, M. B., Cleynhens, K., D'hooge, M., Haelewyck, M. C., De Keyser, J., \& Nagels, G. (2015). Reduced information processing speed as primum movens of cognitive decline in multiple sclerosis. Multiple Sclerosis, 21, 83-91.

Voytek, B., Davis, M., Yago, E., Barceló, F., Vogel, E. K., \& Knight, R. T. (2010). Dynamic neuroplasticity after human prefrontal cortex damage. Neuron, 68(3), 401-408. 